\title{
Nonlinear model matching: a local solution and two worked examples
}

\author{
H.J.C. Huijberts \\ Department of Applied Mathematics, University of Twente, P.O. Box 217, \\ 7500 AE Enschede, The Netherlands
}

\begin{abstract}
The model matching problem consists of designing a compensator for a given system, called the plant, in such a way that the resulting input-output behavior matches that of a prespecified model. In a recent paper it was shown that in case the model is decouplable by static state feedback and generic conditions on the plant are satisfied, the model matching problem is solvable around an equilibrium point if and only if it is solvable for the linearization of plant and model around the equilibrium point. In this paper this local solution will be presented and we will investigate the question to what extent we can use the feedback that solves the corresponding linear model matching problem in order to approximately solve the original nonlinear problem. This will be done by means of two examples: the double pendulum and a two-link robot arm with a flexible joint.
\end{abstract}

\section{Introduction}

A standard approach for dealing with synthesis problems in nonlinear control systems consists in linearizing the nonlinear system around a specific working point and then solving the synthesis problem for the obtained linear system. To what extent the resulting linear controller is a good approximate solution for the nonlinear problem is, of course, questionable.

In the present paper we study this kind of question concerning the so called Model Matching Problem (MMP) for nonlinear systems. This problem is formulated as follows: given a nonlinear control system, to be referred to as the plant $P$, together with another nonlinear system, to be called the model $M$, is it possible to design a suitable precompensator for the plant such that the input-output behavior of the precompensated plant matches that of the given model $M$ ?

The linear model matching problem was completely solved in different set ups by several authors $[5,11,13,14,15]$. For the solution of the nonlinear model matching problem only partial solutions are known up till now (cf. $[9,4,3,7,8,12]$ ).

In [8] it was shown that if plant and model are square systems and the model can be decoupled by static state feedback, then under generic conditions on the plant the problem is solvable around an equilibrium point if and only if it is solvable for the linearization of plant and model.

In this paper we will, following [8], investigate the question whether we can use the feedback that solves the corresponding linear MMP in order to approximately solve the original nonlinear MMP. This investigation will be performed on two examples. First we will consider a double pendulum.
In this example MMP can be solved by means of a static state feedback. Secondly we will consider a two-link robot arm with one flexible joint, as was also studied in e.g. [1]. In this second example we will need a dynamic state feedback to solve MMP. The paper further elaborates the philosophy developed in [6], where similar questions concerning the in putoutput decoupling problem were investigated.

The paper is organized as follows. In the following section we will formulate the nonlinear model matching problem and state our main theorem. This main theorem holds true under generic conditions on the plant $P$. In section 3 we will illustrate the theory by means of the examples mentioned above.

\section{The nonlinear model matching problem}

Consider a nonlinear plant $P$, described by equations of the form:

$$
\begin{aligned}
& \dot{x}=f(x)+\sum_{i=1}^{m} u_{i} g_{i}(x) \\
& y_{i}=h_{i}(x) \quad(i \in\{1, \cdots, m\})
\end{aligned}
$$

with state $x \in \mathcal{X}$, an open neighborhood in $\boldsymbol{R}^{n}$, inputs $u_{i} \in$ $\mathbb{R}$, outputs $y_{i} \in \boldsymbol{R}, f, g_{1}, \cdots, g_{m}$ are real analytic vector fields on $\mathbb{R}^{n}$ and $h_{1}, \cdots, h_{m}$ are real analytic functions.

Furthermore, let a nonlinear model $M$ be given, which is described by the equations:

$$
\begin{aligned}
& \dot{x}^{M}=f^{M}\left(x^{M}\right)+\sum_{i=1}^{m} u_{i}^{M} g_{i}^{M}\left(x^{M}\right) \\
& y_{i}^{M}=h_{i}^{M}\left(x^{M}\right)(i \in\{1, \cdots, m\})
\end{aligned}
$$

with state $x^{N} \in \mathcal{X}^{N}$, an open neigborhood in $\mathbb{R}^{n N}$, inputs $u_{i}^{M} \in \mathbb{R}$, outputs $y_{i}^{M} \in \mathbb{R}, f^{M}, g_{1}^{M}, \cdots, g_{m}^{M}$ are real analytic vector fields on $\mathbb{R}^{n_{N}}$ and $h_{1}^{N}, \cdots, h_{m}^{M}$ are real analytic functions.

Note that for $P$ as well as for $M$ the number of inputs is equal to the number of outputs.

The compensator $Q$ used to control $P$ is a nonlinear system described by equations of the form:

$$
\begin{aligned}
\dot{x}_{c} & =a\left(x_{c}, x\right)+b\left(x_{c}, x\right) u^{\mu} \\
u & =c\left(x_{c}, x\right)+d\left(x_{c}, x\right) u^{N}
\end{aligned}
$$

with state $x_{c} \in \mathcal{X}_{c}$, an open neighborhood in $\mathbb{R}^{\nu}$, and real analytic $a, b, c, d$. The composition of (1) and (3) is denoted by $P \circ Q$, while the output of $P \circ Q$ will be denoted by $y^{P \bullet Q}$.

The usual definition of the model matching problem is given below $($ see $[3,4])$. 
Definition 2.1 Given a plant $P=(f, g, h)$, a model $M=$ $\left(f^{N}, g^{N}, h^{N}\right)$ and a point $\left(x_{0}, x_{0}^{N}\right) \in \mathcal{X} \times \mathcal{X}^{N} \subset \boldsymbol{R}^{n} \times \boldsymbol{R}^{n^{N}}$, the nonlinear model matching problem consists in finding neighborhoods $U$ of $x_{0}$ and $U^{\mu}$ of $x_{0}^{\mu}$, an integer $\nu$, an spen subset $V$ of $\mathcal{X}_{c} \subset R^{\nu}$, a compensator $Q=(a, b, c, d)$ with $u, b, c, d$ renl analytic functions defined on $F \times U$, a map $F: U \times U^{\mathcal{K}} \mapsto V^{r}$, with the property that

$$
y^{P \bullet Q}\left(x, F\left(x, x^{N}\right), t\right)-y^{N}\left(x^{M}, t\right)
$$

is independent of $t^{M \prime}$ for all $t$ and for all $\left(x,:^{M}\right) \in U \times U^{M}$.

Remark 2.2 For linear systems solvability of the $M M P$ comes down to the existence of a (linear) compensitor $Q$ such that the transfer matrices of $M$ and $P \circ Q$ coincide. For nonlinear systems the so-called higher order Volteriv kernels of $M$ and $P \circ Q$ have to coincide.

Define relative degrees $\rho_{1}(x), \cdots, \rho_{m}(x)$ for the plant $P$ via

$$
\rho_{i}(x)=\inf \left\{k \in N \text { s.t. } \mathcal{L}_{g,} \mathcal{L}_{f}^{k-1} h_{i}(x) \neq 0 \text { for some } j\right\}
$$

where $\mathcal{L}_{f} h$ is the directional derivative of the function $h$ along the vector field $f$, i.e. $\mathcal{L}_{f} h(x)=\sum_{i=1}^{\infty} \frac{\partial h}{\partial x_{i}}(x) f_{i}(x)$ and we define inductively: $\mathcal{L}_{f}^{0} h=h, \mathcal{L}_{f}^{k} h=\mathcal{L}\left(\mathcal{L}_{f}^{k-1} h\right)$.

In the same way we define relative degrees $\rho_{i}^{N}\left(x^{N}\right)$ for the model $M$ and $p_{i}^{P \bullet Q}\left(x, x_{c}\right)$ for the compensated plant $P \circ Q$.

The following assumption will be a standing assumption throughout the paper:

(A1) All relative degrees defined above are finite and constant on an open and dense submanifold, i.e.: $\rho_{i}(x)=\rho_{i}<$ $\infty, \rho_{i}^{M}\left(x^{M}\right)=\rho_{i}^{M}<\infty, \rho_{i}^{P \bullet Q}\left(x, x_{c}\right)=\rho_{i}^{P \bullet Q}<\infty$ on an open and dense submanifold

On the open and dense submanifold where (A1) is satisfied the decoupling matrix $A(x)$ of $P$ is defined in the following way:

$$
(A(x))_{i j}=\mathcal{L}_{g_{j}} \mathcal{L}_{f}^{\beta_{i}-1} h_{i}(x) \quad(i, j \in\{1, \cdots, m\})
$$

Similarly we define decoupling matrices $A^{M}, A^{P \bullet Q}$, for $M, P \circ Q$ respectively.

As a second standing assumption we will need:

(A2) The decoupling matrix $A^{M}\left(x^{*}\right)$ has full rank on the open and dense submanifold where it is defined.

For linear systems we have the following (see [11] for a proof):

Proposition 2.3 If $P$ and $M$ are linear systems, the $M M P$ is solvable if and only if

$$
\rho_{i} \leq \rho_{i}^{N} \text { for all } i \in\{1, \cdots, m\}
$$

Recall from [8]:

Proposition 2.4 Consider an analytic plant $P$ and an analytic model M satisfying (A1) and (A2). Then MMP is locally solvable for $(M, P)$ if and only if there is a dymamic compensator $Q$ such that $P \circ Q$ is decouplable by regular static state feedback and $\rho_{i}^{p \circ Q} \leq \rho_{i}^{\mu}$.
It is clear from Proposition 2.4 that in order to solve MMP for a plant $P$ and a model $M$ satisfying (A1i and (A2) we first have to solve the dynamic input-output lecoupling problem for $\boldsymbol{P}$ in some "minimal" way, i.e. we hav $\geq$ to assure that the relative degrees of the compensi.ted plant to not become too large. Rather than pursueing this path, we wili make contact between the solvabilit; of the MMP for $(K, P)$ and the solvability for their lizearization $(L M, L P)$ arc und an equilibrium point, for which Proposition 2.3 gives solvability conditions.

In whas follows it will turn out that we w:ll only address the MMP in a neighborhood of equilibria of model and plant. However, it is rather straightforward that the analyticity of plant and model implies that whenever a local solution of the MMP exists around such an equilibrium, then there exists a local solution around points in an open and dense submanifold of $\mathcal{X} \times \mathcal{X}^{\mathbb{M}}$.

We will first take a closer look at the dynamic input-output decoupling problem and use the algorithm from [16] in order to obtain generic conditions on $P$ under which we can make contact between the solvability of the MMP for $(M, P)$ and the solvability for $(L M, L P)$.

The problem of dynamic input-output decoupling was studied in e.g. $[2,16,18]$. Essentially the algorithm of $[16]$ (as well as the other algorithms do) works on an open and dense submanifold of points $\left(x, x_{c}\right)$ in $\mathcal{X} \times \mathcal{X}_{c}$. Our basic assumption will be that given the equilibrium point $I_{0}$ for $P$ there exists an equilibrium point $\left(x_{0}, x_{\infty}\right)$ for the precompensated system $P \circ Q$ at which the algorithm can be effectively applied. To make this more transparent we shortly discuss the first step of the algorithm of [16]. Given the plant $P$ we compute its decoupling matrix $A(x)$ according to equation (6). Clearly the rank of $A(x)$ is constant on an open and dense submanifold $\mathcal{X}^{1}$ of $\mathcal{X}$. Assume $x_{0} \in \mathcal{X}^{1}$. Next, a regular static state feedback

$$
u=\alpha(x)+\beta(x) \bar{u}
$$

is applied to $P$, and which leaves the equilibrium point $x_{0}$ invariant. Note that applying such a feedback does not change the rank of the decoupling matrix $A(z)$. The first step of the algorithm proceeds with adding a bank of integrators $\dot{\nu}_{i 1}=$ $z_{i 2}, \cdots, \dot{z}_{i k_{i}}=v_{i}, \tilde{u}_{i}=z_{i 1}$ for $i=1, \cdots, m$ and certain integers $k_{i} \in N$. Obviously, adding (8) and a bank of integrators to $P$ yields a system on $\mathcal{X} \times \mathcal{Z}$, where $\mathcal{Z}=\left(z_{11}, \cdots, z_{1 k_{1}}, \cdots, z_{m 1}\right.$, $\left.\cdots, z_{m k_{m}}\right)$ and this system possesses an equilibrium point $\left(x_{0}, z_{0}\right) \in \mathcal{X} \times \mathcal{Z}$. We now compute the decoupling matrix of $P$ together with (8) and the bank of integrators, to be denoted as $A(x, z)$. Again this decoupling matrix has constant rank on an open and dense submanifold $(\mathcal{X} \times \mathcal{Z})^{1}$ of $\mathcal{X} \times \mathcal{Z}$. We assume $\left(x_{0}, x_{0}\right)$ belongs to $(\mathcal{X} \times \mathcal{Z})^{1}$. The algorithm of [16] now repeats the above steps of applying state feedbacks (8) and adding a bank of integrators. Moreover the algorithm terminates after a finite number of steps. Our basic assumption can now be stated as follows:

(A3) Consider the system (1) around the equilibrium point $x_{0} \in \mathcal{X}$. Then we assume that at each step of the above algorithm the decoupling matrix $A(x, z)$ has constant rant on a neighborhood of the equilibrium point $\left(x_{0}, z_{0}\right)$.

Essentially (A3) says that the algorithm of [16] can be applied on a neighborhood of the equilibrium point $x_{0}$. Given the 
analyticity of (1), this condition is generically met for vector fields $f, g_{1}, \cdots, g_{m}$ and functions $h_{1}, \cdots, h_{m}$.

We now come to the statement of our main result.

Theorem 2.5 Consider an analytic plant $P$ and an analytic model $M$ satisfying (A1) and (A2). Let $x_{0}$ and $x_{0}^{*}$ be equilibrium points of respectively $P$ and $M$. Assume that $P$ satisfies (A3). Let $L P, L M$ denote the linearizations of $P$ around $x_{0}$ and $M$ around $x_{0}^{*}$ respectively. Then the $M M P$ is locally solvable for $(M, P)$ if and only if it is solvable for $(L M, L P)$.

Proof See [8].

\section{Examples}

In this section we will investigate the question to what extent we can use the feedback that solves the corresponding linear MMP as an approximate solution of the original nonlinear MMP by means of two examples.

\subsection{The double pendulum}

In this example our plant will be a double pendulum consisting of two rigid massless links of unit length, with two unit masses attached at the ends of the links. As configuration variables we take $\theta_{1}$, the angle between the first link and a line perpendicular to the base, and $\theta_{2}$, the angle between the first and second link. As inputs we take control torques $u_{1}$ and $u_{2}$, that are applied at each joint. As outputs we take $y_{1}=\theta_{1}, y_{2}=\theta_{2}$. The Euler-Lagrange equations of motion for this system read:

$$
M(\theta) \ddot{\theta}+P(\theta, \dot{\theta})+Q(\theta)=u
$$

with $u=\left(u_{1}, u_{2}\right)^{T}, \theta=\left(\theta_{1}, \theta_{2}\right)^{r}$, and

$$
\begin{aligned}
M(\theta) & =\left[\begin{array}{cc}
3+2 \cos \theta_{2} & 1+\cos \theta_{2} \\
1+\cos \theta_{2} & 1
\end{array}\right] \\
P(\theta, \dot{\theta}) & =\left[\begin{array}{c}
\sin \theta_{2}\left(2 \dot{\theta}_{1} \dot{\theta}_{2}+\dot{\theta}_{2}^{2}\right) \\
\dot{\theta}_{2}^{2} \sin \theta_{2}
\end{array}\right] \\
Q(\theta) & =\left[\begin{array}{c}
20 \sin \theta_{1}+10 \sin \left(\theta_{1}+\theta_{2}\right) \\
10 \sin \left(\theta_{1}+\theta_{2}\right)
\end{array}\right]
\end{aligned}
$$

where we have put the constant of gravity $g=10$.

This system has relative degrees $\rho_{1}=\rho_{2}=2$, so (A1) is satisfied for the plant. The decoupling matrix of the system is just the matrix $M(\theta)$, which can easily be proved to have full rank for all $\theta$ and hence (A3) is satisfied.

As a model we take a stable decoupled linear time-invariant system with transfer matrix given by: $G^{M}(s)=\operatorname{diag}((s+$ $\left.3)^{-2},(s+3)^{-2}\right)$ It follows immediately that the model has relative degrees $\rho_{1}^{M}=\rho_{2}^{M}=2$ and that its decoupling matrix has full rank. Hence the model satisfies (A1) and (A2) and furthermore it can easily be checked that the model matching problem is solvable.
The control that solves MMP for the linearization of the plant around $\left(\theta_{1}, \theta_{2}, \dot{\theta}_{1}, \dot{\theta}_{2}\right)=(0,0,0,0)$ and the given model is given by:

$$
u=F\left[\begin{array}{l}
\theta \\
\dot{\theta}
\end{array}\right]+G u^{*}
$$

with $F=\left[\begin{array}{cccc}-15 & -30 & 42 & -12 \\ -8 & -12 & 21 & -6\end{array}\right], G=\left[\begin{array}{ll}5 & 2 \\ 2 & 1\end{array}\right]$.

Figures 2 and 3 give the results of simulations of the system (9) with the control (10). In figure 2 a step $t_{1}^{N}=1$ is applied, while in figure 3 a step $u_{2}^{\mu}=1$ is applied. These steps are applied during the whole time-interval. The figures give the errors $e_{i}(t)=y_{i}(t)-y_{i}^{N}(t)(i=1,2)$.

The system converges to a steady state situation with steady state errors $e_{1}=-32 \cdot 10^{-6}(0.02 \%), e_{2}=-258 \cdot 10^{-6}$ in the first case and $e_{1}=0.1290, e_{2}=0.0067(6.1 \%)$ in the second case. Hence in the first case our linear control behaves reasonably well, while in the second case the results are less satisfying.

The fact that we end up with a steady state error suggests that we can improve the behavior of the system by including a copy of the model in the compensator and then applying an extra PI-action. Figure 4 shows that this indeed works. Here we have again applied a step $u_{2}^{N}=1$, while to $u_{1}$ in (10) we have added a term $-12.8 e_{1}(t)-10 \int_{0}^{t} e_{1}(\tau) d \tau$ and to $u_{2}$ in (10) we have added a term $-10 \int_{0}^{t} e_{2}(\tau) d \tau$. We now end up with a steady state error $e_{1}=0.0014$ ( $1 \%$ of the steady state error in figure 3$)$ and $e_{2}=242 \cdot 10^{-6}(0.22 \%$ of the steady state value of $\left.y_{2}^{M}\right)$.

Figure 5 shows a phase portrait in the $\left(\theta_{1}, \theta_{2}\right)$-plane of the system (9) with the control (10) and the PI-control mentioned above when a step $u_{1}^{N}=1, u_{2}^{\mu}=1$ is applied. The steady state situation is reached after about 25 seconds. The nominal trajectory is a straight line from the origin to the point $\left(\theta_{1}, \theta_{2}\right)=(0.1111,0.1111)$. The steady state errors equal $e_{1}=0.0015(1.33 \%)$ and $e_{2}=111 \cdot 10^{-6}(0.1 \%)$.

Further simulations (of which no figures are included) show that the stability of the closed loop system is maintained for step inputs up to $\left(u_{1}^{N}, u_{2}^{N}\right)=(8,0),\left(u_{1}^{N}, u_{2}^{M}\right)=(0,3)$, $\left(u_{1}^{N}, u_{2}^{N}\right)=(2,2)$. In the first two case (i.e. if we take one of the inputs equal to zero) the maximum errors and steady state errors are proportional to the magnitude of the input.

\subsection{Robot arm with flexible joint}

As a second example we consider a two link robot arm moving in a vertical plane, as was described in e.g. [1]. The first link is actuated through a direct drive motor. The rotation of the first link with respect to a line perpendicular to the base is indicated by $q_{1}$. The second joint shows a significant elasticity. This elasticity is modelled by associating two variables to the second joint: $q_{2}$, the position of the second actuator with respect to the first link and $q_{3}$, the position of the second link with respect to the first one. The motor $\left(q_{2}\right)$ is then coupled to the joint $\left(q_{3}\right)$ by means of a transmission with transmission ratio $N T>1$ and a torsional spring with spring constant $K$ (see figure 1 ). In this example we will have links of unit length, motors with inertia equal to 0.001 and we will assume 
the masses to be equal to one and to be concentrated at the joints (the motors) and at the tip (a load). Furthermore ve will have $K=1000, N T=100$ and the constant of gravity $g=10$.

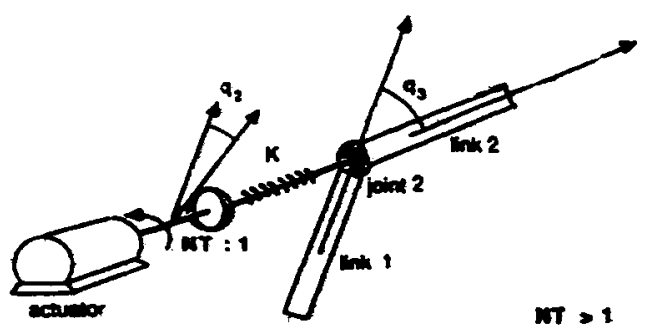

Figure 1: Definition of variables and parameters for elabic joint

The Duler-Iagrato equations of motion for this system read:

$$
M(\varphi) \bar{y}+N(\varphi, \phi)=\omega_{5}
$$

$$
\text { with } g=\left(n, n_{1}, s_{3}\right)^{s}, w_{E}=\left(v_{1}, v_{2}, 0\right)^{s} \text { and }
$$

$$
\begin{aligned}
& M(q)=\left[\begin{array}{ccc}
3.002+2 \cos 3 & 0.001 & 1+\cos s \\
0.001 & 0.001 & 0 \\
1+\cos \theta & 0 & 1
\end{array}\right]
\end{aligned}
$$

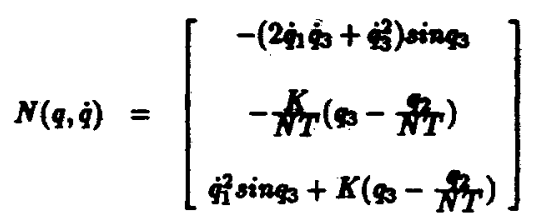

We will take as ontputs $y_{1}=q_{1}, y_{2}=q_{3}$. This system has relative degrees $\rho_{1}=\rho_{2}=2,80$ (A1) is satisfied for the plant. Furthermore it can be checked that also (A3) is satisfied for the plant (see [1] for details).

As a model we take a stable decoupled linear time-invariant system with transfer matrix given by $G^{M}(s)=\operatorname{diag}(s+$ $\left.3)^{-4},(s+3)^{-4}\right)$. It follows immediately that the model has relative degrees $\rho_{1}^{N}=\rho_{2}^{N}=4$ and that its decoupling matrix has full rank. Hence the model satisfies (A1) and (A2). It can easily be chected that MMP is solvable for the linearizations of the plant and the model around an equilibrium point and hence MMP is solvable for the original nonlinear systems.

A contral law that solves MMP for the linearization of the plant around $(g, \dot{q})=(0,0)$ and the model is given by:

$$
\begin{aligned}
& \dot{x}_{c}=K x_{c}+L\left[\begin{array}{l}
q \\
\dot{q}
\end{array}\right]+M u^{N} \\
& {\left[\begin{array}{l}
v_{1} \\
v_{2}
\end{array}\right]=R\left[\begin{array}{c}
q \\
\dot{1} \\
x_{c}
\end{array}\right]+S u^{\mu}}
\end{aligned}
$$

witl

$$
\begin{aligned}
& x_{c}=\left(x_{e 1}, x_{e 2}\right)^{T} \\
& K=\left[\begin{array}{cc}
0 & 1 \\
-54 & -12
\end{array}\right] \\
& L=\left[\begin{array}{cccccc}
0 & 0 & 0 & 0 & 0 & 0 \\
-81 & 0 & 0 & -16 & 0 & 0
\end{array}\right] \\
& M=\left[\begin{array}{l}
0 \\
1
\end{array}\right] \\
& \boldsymbol{R}=\left[\begin{array}{cccc}
9.0278 & 20.956 & -2106.5641 & -0.0096 \\
-0.9722 & 1.056 & -106.5611 & -0.0006
\end{array}\right. \\
& \left.\begin{array}{cccc}
-0.012 & 1.2012 & -0.2 & 0 \\
-0.012 & 1.2012 & -0.2 & 0
\end{array}\right] \\
& S=\left[\begin{array}{ll}
0.0002 & 0.0001 \\
0.0002 & 0.0001
\end{array}\right]
\end{aligned}
$$

Figures 6 and 7 give the results of simulations of the system (11) with the control (12). In figure 6 a step $x_{1}^{\prime \prime}=1$ is applied, while in figure 7 a step $=4$ is applied. The steps are applied during the whole time period. The figures give the errors $c_{i}(t)=n_{i}(t)-n_{i}^{*}(t)(i=1,2)$.

In the first case the system converges again to a steady state situation with steady state errors $e_{1}=2 \cdot 10^{-6}(0.02 \%), e_{2}=$ $35 \cdot 10^{-6}$. In the second case we end up with luctuations aroand $e_{1}=-2 \cdot 10^{-6}, e_{2}=-102 \cdot 10^{-6}(0.82 \%)$.

In figures 8 and 9 it is shown that we can again improve the behavior of the system by including a copy of the model in the compensator and then applying an extra PI-action. In this case we have added $a$ term $-\int_{0}^{t} e_{1}(\tau) d \tau$ to $\Sigma_{1}$ in (12) and a term $-0.00195 \int_{0}^{t} e_{2}(\tau) d \tau$ to $y_{2}$ in (12). In figure 8 we have applied a step $v_{1}^{N}=1$, while in figure 9 we have applied a step $u_{2}^{\mu}=1$. In the first case the steady state errors become $e_{1}=-5 \cdot 10^{-9}, e_{2}=706 \cdot 10^{-9}$, and in the second case $e_{1}=$ $29 \cdot 10^{-9}, e_{2}=597 \cdot 10^{-9}$.

Further simulations show that the stability of the closed loop system is maintained for step inputs up to $\left(u_{1}^{*}, u_{2}^{*}\right)=(12,0)$, $\left(w_{1}^{N}, u_{2}^{*}\right)=(0,9),\left(z_{1}^{*}, w_{2}^{*}\right)=(5,5)$.

\section{Conclusions}

In this paper we have presented a local solution of the nonlinear model matching problem. Furthermore by means of two examples we have investigated the question whether we can use the compensator that solves the MMP for the linearization of plant and model around an equilibrium point in order to approximately solve the original nonlinear MMP around the 
same equilibrium point.

For both examples the linear feedback worked reasonably well, except in the first example, when we apply a step on the second model input. However, the performance of the control can be considerably improved by introducing an extra PI-action. It is to be expected that the introduction of an extra D-action will further improve the performance.

In this paper we have restricted ourselves to models of which the decoupling matrix has full rank, i.e. models that can be decoupled by static state feedback. This assumption is certainly restrictive, but from a practical point of view it can be argued that it is often desirable.

\section{Acknowledgments}

I would like to thank Henk Nijmeijer for the many useful discussions we have had on the organization and contents of this paper.

\section{References}

1. De Luca, A. (1988). Control properties of robot arms with joint elasticity. In C.I. Byrnes, C.F. Martin and R.E. Saeks (Eds.), Analysis and control of nonlinear systems. Elsevier, Amsterdam. pp. 61-70.

2. Descusse, J., and C. Moog (1985). Decoupling with dynamic compensation for strong invertibility affine nonlinear systems. Int. J. Control, 42, 1387-1398.

3. Di Benedetto, M.D., (1988). A condition for the solvability of the nonlinear model matching problem. In J. Descusse, M. Fliess, A. Isidori and D. Leborgne (Eds.) New trends in nonlinear control theory, Lecture notes in control and information sciences 122. Springer, Berlin. pp. 102-115.

4. Di Benedetto, M.D. and A. Isidori (1986). The matching of nonlinear models via dynamic state feedback. SIAM J. Control Optimiz., 24, 1063-1075.

5. Emre, E. and M.L.J. Hautus (1980). A polynomial characterization of (A,B)-invariant and reachability subspaces. SIAM J. Control Optimiz., 18, 420-436.

6. Gras, L.C.J.M., and H. Nijmeijer (1989). Decoupling in nonlinear systems: from linearity to nonlinearity. IEE Proceedings, 136, Pt. D., 53-62.

7. Huijberts, H.J.C. (1989). Nonlinear model matching, with an application to Hamiltonian systems. Preprints IFAC Non linear control systems design symposium 1989, Capri, 319-324.

8. Huijberts, H.J.C. and H. Nijmeijer (1989). Local nonlinear model matching: from linearity to nonlinearity. To appear in Automatica 26 (6).

9. Isidori, A. (1985). The matching of a prescribed linear input-output behavior in a nonlinear system. IEEE Trans. Aut. Control, AC-30, 258-265.

10. Isidori, A. (1989). Nonlinear control systems (2nd Edition). Springer, Berlin.
11. Malabre, M. (1982). Structure à l'infini des triplets invariants. Application à la poursuite parfaite de modèle. In A. Bensoussan and J.L. Lions (Eds.), Analysis and optimization of systems, Lecture notes in control and information sciences, Vol. 44. Springer, Berlin. pp. 43-53.

12. Moog, C.H., A.M. Perdon and G. Conte (1989). Model matching and factorization for nonlinear systems: a structural approach. To appear in SIAM J. Control Optimiz..

13. Morse, A.S. (1973). Structure and Design of Linear Model Following Systems. IEEE Trans. Aut. Control, AC-18, 346-354.

14. Morse, A.S. (1976). Minimal solutions to transfer matrix equations. IEEE Trans. Aut. Control, AC-21, 131-133.

15. Moore, B.C. and L.M. Silverman (1972). Model matching by state feedback and dynamic compensation. IEEE Trans. Aut. Contr., AC-17, 491-497.

16. Nijmeijer, $H$. and W. Respondek (1988). Dynamic Input-Output Decoupling of Nonlinear Control Systems. IEEE Trans. Aut. Contr., AC-33, 1065-1070.

17. Silverman, L.M. (1969). Inversion of multivariable linear systems. IEEE Trans. Aut. Control, AC-14, 270-276.

18. Singh, S.N. (1980). Decoupling of invertible nonlinear systems with state feedback and precompensation. IEEE Trans. Aut. Control, AC-25, 1237-1239.
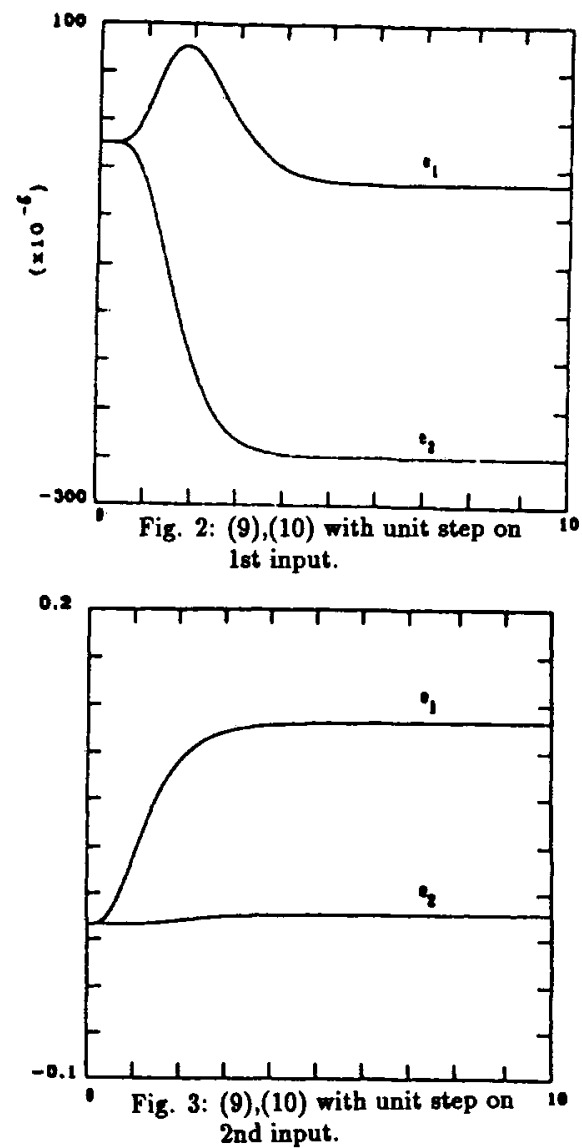


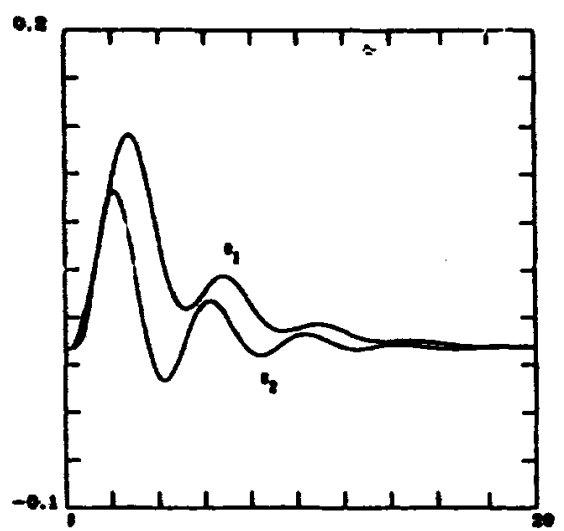

Fig. 1: (9),(10), Pl-control with unit atep on 2nd input.

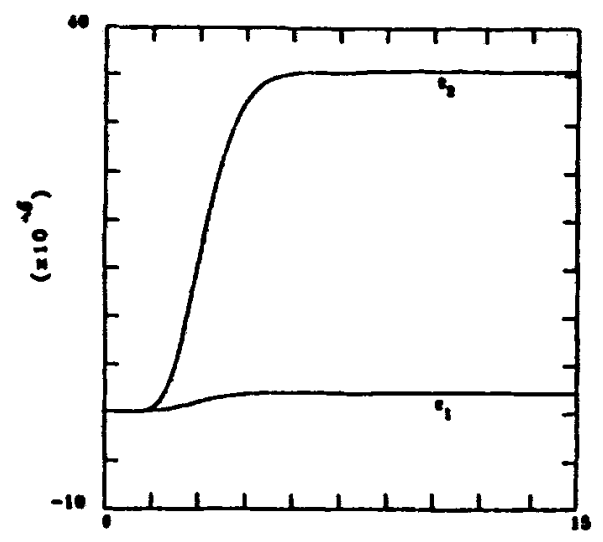

Fig. 6: (11),(12) with unit tep on lat inpet.

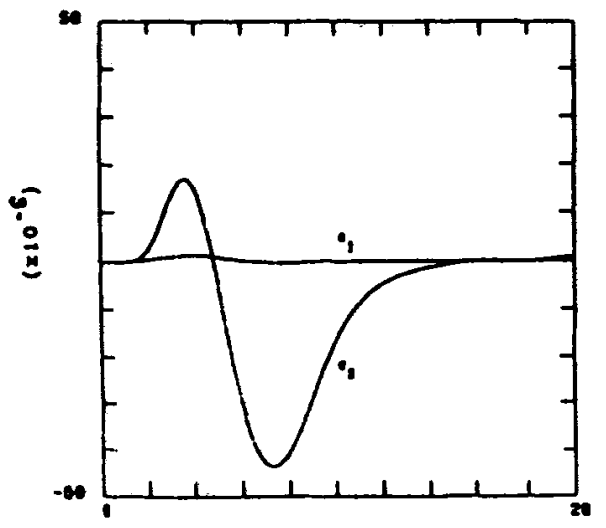

Fig. 8: (11),(12),PL-control with unit step on let input.

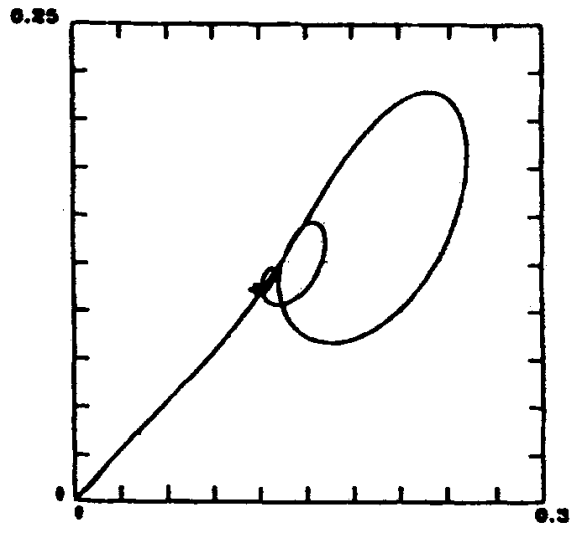

Fig. 5: Pl-control and unit atep on lst and 2nd input.

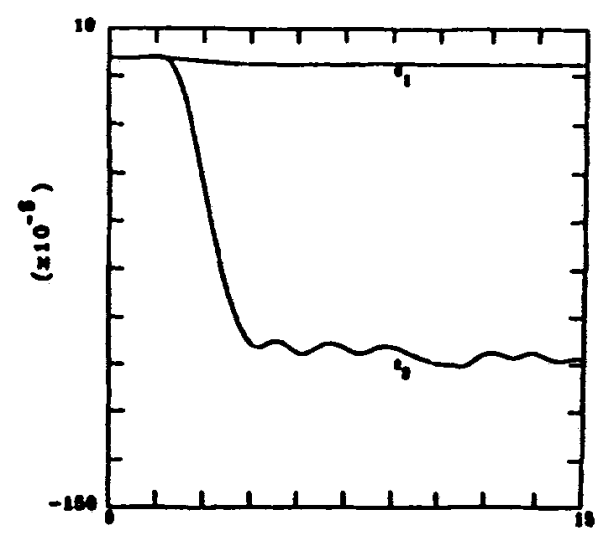

Fig. 7: (11),(12) with unit step on 2nd inpat.

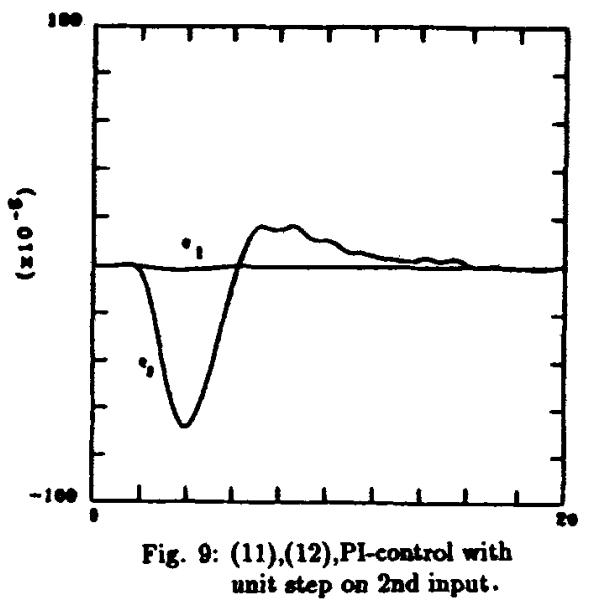

